\title{
Ischemic Mitral Regurgitation
}

\author{
Hirofumi Takemura
}

\begin{abstract}
Ischemic mitral regurgitation (MR) is defined as functional MR caused by myocardial ischemia. Apical and posterior displacement of papillary muscles, referred to as tethering, positively correlates with maximal regurgitation area. Surgical intervention for patients with moderately ischemic MR at the time of coronary artery bypass (CABG) remains controversial. However, patients with uncorrected mild or moderate MR who undergo $\mathrm{CABG}$ are at increased risk of death and hospitalization. Restrictive MV annuloplasty and coronary artery bypass surgery has become accepted as the standard technique for severe functional MR. However, even after downsizing simple annuloplasty by two sizes, MR persists or recurs in $>20 \%$ of patient. Furthermore, recurrent and progressive MR after MV plasty is associated with high mortality rates. Predictors for recurrent mitral regurgitation are flexible ring usage, left ventricle diameter, continuous LV remodeling, sphericity index at endsystole and end-diastole, and preoperative posterior leaflet angle $>45^{\circ}$. Second chordal cutting, papillary muscle approximation and so on are recommended as additional procedures. Whether to replace or repair severe chronic ischemic MV regurgitation has remained controversial. Replacement results in long-term secure MV sufficiency, whereas mitral repair still confers some risk of MR recurrence. Percutaneous MV repair using the MitraClip procedure can offer clinical improvement even in patients with high surgical risk and severe functional MR. The long-term outcomes of the this procedure should be determined. Ischemic MR remains a significant complication of myocardial infarction, and deeper understanding of the mechanism of ischemic MR has led to continuous improvements in surgical treatment. Complex mechanisms involving the mitral annulus, chordae tendinea, papillary muscle, and LV are involved, and optimal surgical repair should consider the overall pathology of each patient.
\end{abstract}

KEY WORDS: functional mitral regurgitation, ischemic mitral regurgitation, left ventricular remodeling, papillary muscle, tethering

\section{Definition and mechanism of ischemic mitral regurgitation}

Ischemic mitral regurgitation (MR) is defined as functional MR caused by myocardial ischemia including myocardial infarction and ischemic cardiomyoplasty that results in disturbed left ventricular (LV) geometry. It does not usually coexist with rheumatic heart valve disease, degenerative disease, or congenital defect of the mitral valve (MV) ${ }^{1}$ and is associated with excess mortality independently of baseline characteristics and the severity of ventricular dysfunction ${ }^{2)}$. The mechanism of regurgitation is independently multifactorial, including LV dilation, LV sphericity, apical and posterior displacement of the papillary muscle, and LV function. Apical and posterior displacement, referred to as tethering, positively correlates with maximal regurgitation $\operatorname{area}^{1,3)}$.

Department of Thoracic, Cardiovascular and General Surgery, Kanazawa University, 13-1 Takara-machi, Kanazawa, Ishikawa 920-8641, Japan DOI: $10.7793 /$ jcad.25.003

\section{Moderate mitral regurgitation at coronary artery bypass surgery}

That severe ischemic MR should be corrected has reached consensus among surgeons and physicians, but surgical intervention for patients with moderately ischemic MR at the time of coronary artery bypass $(\mathrm{CABG})$ remains controversial, However, a comparison of long-term results after $\mathrm{CABG}$ according to $\mathrm{MR}$ grade evaluated by intraoperative $\mathrm{TEE}^{4)}$ found that even mild MR predicts worse outcomes after CABG. The report of that study emphasized that patients with uncorrected mild or moderate MR who undergo CABG are at increased risk of death and hospitalization for heart failure, leading to consideration for further surgical repair or more aggressive medical management. Others have also found that even preoperative moderate grade of MR that improves immediately after $\mathrm{CABG}$ worsens during the late phase ${ }^{5,6)}$.

On the other hand, preoperative moderate ischemic MR can be relieved by off-pump CABG in selected patients ${ }^{7)}$. The predictors are ejection fraction $>37.1 \%$, posteroinferior volume ra- 
tio $>31.6 \%$, and surgery $<3$ months after myocardial infarction Penicka et al. also found that moderate MR improved after isolated CABG only if patients had concomitant viable myocardium and no dyssynchrony between papillary muscles ${ }^{8}$. Fattouch et al. concluded that papillary muscle relocation plus nonrestrictive mitral annuloplasty at the time of $\mathrm{CABG}$ might promote a significant reversal in LV remodeling, with a considerable decrease in the tenting area and coaptation depth ${ }^{9}$. Chan et al. concluded from the randomized control RIMA study that adding mitral annuloplasty to CABG for patients with moderately ischemic MR might improve functional capacity, LV reverse remodeling, MR severity, and B-type natriuretic peptide values more effectively than CABG alone ${ }^{10)}$. Bouchard et al. concluded from another randomized control study that although initially effective at reducing moderate functional ischemic $\mathrm{MR}$, adding a ring did not change the clinical course after CABG surgery and an echocardiographic difference was not identified in terms of residual mitral regurgitation, LV dimensions and function, or clinical outcomes at 12 months $^{11)}$.

In contrast, Kang et al. did not find any difference between additional MV repair and CABG alone, and considered that the latter might be a preferable treatment option for patients with moderate MR and high operative risk factors such as advanced age or atrial fibrillation ${ }^{12}$. Altarabsheh et al. concluded from a systemic review and meta-analysis that concomitant repair of moderate ischemic MR leads to improved MV competence at follow-up, whereas adding valve repair to CABG did not translate into a functional or survival benefit at five years of follow-up ${ }^{13)}$. Another study showed that adding repair or replacement to CABG in patients with moderately ischemic MR did not result in improved early or overall mortality, stroke risk, or intermediate markers of LV function compared with CABG alone ${ }^{14)}$.

\section{How and what to repair}

Although the methods of treating degenerative MV disease have reached general consensus, the management of ischemic MR remains controversial. Restrictive MV annuloplasty and coronary artery bypass surgery, which had mostly been applied to treat cardiomyopathy, has become accepted as the standard technique for functional $\mathrm{MR}^{15)}$. However, even after downsizing simple annuloplasty by two sizes, MR persists or recurs in $>20 \%$ of patients ${ }^{16)}$. Furthermore, recurrent and progressive MR after MV plasty is associated with high mortality rates. Crabtree et al. found better long-term survival rates after MV repair among patients with 0 to $2+$ than $3+$ to $4+$ MR. However, MR progresses despite repair in many patients and it is associated with poorer survival rates ${ }^{17)}$.

\section{Predictors of MR recurrence}

\section{Flexible versus rigid rings}

Ischemic MR can be corrected in selected patients using restrictive annuloplasty with either flexible or rigid annular rings. Flexible rings are associated with an increased likelihood of MR recurrence that will require reoperation, whereas rigid rings appear to significantly reduce the need for repeat surgical procedures $^{18,19)}$

\section{LV dysfunction}

Braun et al. compared long-term outcomes according to LV diameter cutoff. They concluded that intermediate-term cutoff values for LV reverse remodeling at 4.3 years of follow-up were predictors of later mortality. Restrictive mitral annuloplasty with revascularization for patients with preoperative LV end diastolic diameter $\leq 65 \mathrm{~mm}$ can cure ischemic mitral regurgitation and heart failure $^{20)}$

\section{Continuous LV remodeling}

Hung reviewed serial echoes in 30 patients with recurrent MR versus early after ring annuloplasty (RA) for ischemic $\mathrm{MR}^{21)}$. That review showed that even if ischemic MR is corrected immediately after mitral ring repair, LV remodeling, that is, progression with increasing LV volume and sphericity index at end-systole and end-diastole, continues. Kuwahara et al. investigated the characteristics of leaflet configurations long after postoperative MR in 30 patients who underwent annuloplasty for ischemic $M^{22}$. They found significantly worse posterior than anterior tethering of mitral leaflets soon after surgery. In addition, posterior tethering became progressively worse in patients with late MR recurrence. Magne showed that patients with a preoperative posterior leaflet angle $>45^{\circ}$ should be considered poor candidates for $\mathrm{RA}^{23)}$.

\section{Additional procedures}

\section{Second chordal cutting}

Messas showed that second-order strut chordae that insert symmetrically into the central ventricular surfaces of both leaflets pull the leaflets down into the ventricle with considerable leaflet distortion that resembles the shape of a seagull. They showed that cutting secondary chordae limits progressive increases in LV volume ${ }^{24-26)}$. Borger et al. reported clinical findings showing that cutting the chordae improves the mobility of MV leaflets and reduces mitral regurgitation recurrence without obvious deleterious effects on LV function ${ }^{27)}$. Calafiore et al. reported that cutting the chordae is related to lower incidence of MR return or persistence, improved EF, and a lower New York Heart Association class in selected patients with an anterior leaflet angle $>145^{\circ}$ and a coaptation depth $<10 \mathrm{~mm}^{28)}$. 


\section{Papillary muscle approximation}

Hvass et al. developed a new functional approach to mitral repair for ischemic LV dysfunction and functional MR. They described that the position of the anterior papillary muscle (PM) is usually normal, whereas the posterior PM is dislocated medially. Passing a 4-mm Gore-Tex tube around both PM causes the posterior PM to become closer to the anterior PM. Postoperative echocardiography revealed that the tenting effect decreased from 14 to $1.4 \mathrm{~mm}^{29}$. . Rama et al. also described a similar technique ${ }^{30)}$. Kron relocated the posterior PM close to the posterior annulus for effective coaptation of the leaflet ${ }^{31}$. Langer and Arai et al. changed the relocation direction towards the anterior annulus ${ }^{32,33)}$ and Nappi et al. concluded that RA + papillary muscle approximation exerted a long-term beneficial effect on LV remodeling and more effectively restored the MV geometric configuration compared with RA alone, but overall mortality and quality of life did not significantly differ between the two procedures ${ }^{34)}$.

\section{MVR or MVP or MitraClip}

Whether to replace or repair severe chronic ischemic MV regurgitation has remained controversial. Replacement results in long-term secure MV sufficiency, whereas mitral repair still confers some risk of MR recurrence. Although surgical techniques for MV repair have evolved as noted above, continuous LV remodeling resulting in recurrent valve incompetence has remained a major downside of this approach. The tradeoff is between the durability of MV repair to correct a regurgitant valve and the adverse consequences of prosthetic valve insertion. Lorusso et al. concluded that MV replacement is a suitable option for patients with chronic ischemic MR and impaired LV function. This strategy provides better outcomes in terms of freedom from reoperation with comparable valve-related complication rates. However, MV repair remains the most powerful predictor of a need for MV re-operation ${ }^{35,36)}$. The randomized CSTN trial found that mid-term survival and LV reverse remodeling did not significantly differ between restrictive annuloplasty repair and chordal-sparing valve replacement in patients with severely ischemic MR, but the incidence of recurrent MR and heart failure complications was greater after repair ${ }^{37}$. Matsui proposed that MV replacement preservation and further approximation of the PM might be more effective ${ }^{38)}$.

\section{MitraClip procedure}

Percutaneous MV repair using the MitraClip procedure can offer clinical improvement even in patients with high surgical risk and severe functional MR. Giannini et al. assessed the survival rates and clinical outcomes of severely ischemic MR after optimal medical therapy (OMT) compared with those treated with a MitraClip. They demonstrated better overall survival as well as freedom from cardiac death and readmission due to car- diac disease when patients were treated with this device than with $\mathrm{OMT}^{39}$. Mendirichaga et al. reviewed 12 studies including 1,695 patients with moderate or severe functional MR and high surgical risk. The rate of mitral reintervention at 12 months was $3 \%$, indicating the feasibility and safety of transcatheter MV treatment using a MitraClip ${ }^{40)}$. The long-term outcomes of the MitraClip procedure should be determined.

\section{Conclusions}

Ischemic MR remains a significant complication of myocardial infarction, and deeper understanding of the mechanism of ischemic MR has led to continuous improvements in surgical treatment. Complex mechanisms involving the mitral annulus, chordae tendinea, papillary muscle, and LV are involved, and optimal surgical repair should consider the overall pathology of each patient. Repair or replacement, as well as whether moderate ischemic MR should be addressed by coronary revascularization alone at the time of CABG and the type of subvalvular manipulation remain matters of debate.

\section{Conflicts of interest}

None.

\section{References}

1) Magne J, Sénéchal M, Dumesnil JG, et al: Ischemic mitral regurgitation: a complex multifaceted disease. Cardiology 2009; 112: 244 259

2) Grigioni F, Enriquez-Sarano M, Zehr KJ, et al: Ischemic mitral regurgitation: long-term outcome and prognostic implications with quantitative Doppler assessment. Circulation 2001; 103: 1759-1764

3) Nagasaki M, Nishimura S, Ohtaki E, et al: The echocardiographic determinants of functional mitral regurgitation differ in ischemic and non-ischemic cardiomyopathy. Int J Cardiol 2006; 108: 171-176

4) Schroder JN, Williams ML, Hata JA, et al: Impact of mitral valve regurgitation evaluated by intraoperative transesophageal echocardiography on long-term outcomes after coronary artery bypass grafting. Circulation 2005; 112 (9 Suppl): I293-298

5) Goland S, Czer LS, Siegel RJ, et al: Coronary revascularization alone or with mitral valve repair: outcomes in patients with moderate ischemic mitral regurgitation. Tex Heart Inst J 2009; 36: 416-424

6) Mihaljevic T, Lam BK, Rajeswaran J, et al: Impact of mitral valve annuloplasty combined with revascularization in patients with functional ischemic mitral regurgitation. J Am Coll Cardiol 2007; 49: 2191-2201

7) Sun X, Huang J, Shi M, et al: Predictors of moderate ischemic mitral regurgitation improvement after off-pump coronary artery bypass. J Thorac Cardiovasc Surg 2015; 149: 1606-1612

8) Penicka M, Linkova H, Lang O, et al: Predictors of improvement of unrepaired moderate ischemic mitral regurgitation in patients undergoing elective isolated coronary artery bypass graft surgery. Circulation 2009; 120: 1474-1481

9) Fattouch K, Castrovinci S, Murana G, et al: Papillary muscle reloca- 
tion and mitral annuloplasty in ischemic mitral valve regurgitation: midterm results. J Thorac Cardiovasc Surg 2014; 148: 1947-1950

10) Chan KM, Punjabi PP, Flather M, et al: Coronary artery bypass surgery with or without mitral valve annuloplasty in moderate functional ischemic mitral regurgitation: final results of the Randomized Ischemic Mitral Evaluation (RIME) trial. Circulation 2012; 126: $2502-$ 2510

11) Bouchard D, Jensen $H$, Carrier M, et al: Effect of systematic downsizing rigid ring annuloplasty in patients with moderate ischemic mitral regurgitation. J Thorac Cardiovasc Surg 2014; 147: 1471-1477

12) Kang DH, Kim MJ, Kang SJ, et al: Mitral valve repair versus revascularization alone in the treatment of ischemic mitral regurgitation. Circulation 2006; 114 (1 Suppl): I499-503

13) Altarabsheh SE, Deo SV, Dunlay SM, et al: Meta-analysis of usefulness of concomitant mitral valve repair or replacement for moderate ischemic mitral regurgitation with coronary artery bypass grafting. Am J Cardiol 2017; 119: 734-741

14) Anantha Narayanan M, Aggarwal S, Reddy YNV, et al: Surgical repair of moderate ischemic mitral regurgitation-A systematic review and meta-analysis. Thorac Cardiovasc Surg 2017; 65: 447-456

15) Bolling SF, Pagani FD, Deeb GM, et al: Intermediate-term outcome of mitral reconstruction in cardiomyopathy. J Thorac Cardiovasc Surg 1998; 115: 381-386; discussion 387-388

16) McGee EC, Gillinov AM, Blackstone EH, et al: Recurrent mitral regurgitation after annuloplasty for functional ischemic mitral regurgitation. J Thorac Cardiovasc Surg 2004; 128: 916-924

17) Crabtree TD, Bailey MS, Moon MR, et al: Recurrent mitral regurgitation and risk factors for early and late mortality after mitral valve repair for functional ischemic mitral regurgitation. Ann Thorac Surg 2008; 85: 1537-1542; discussion 1542-1543

18) Spoor MT, Geltz A, Bolling SF: Flexible versus nonflexible mitral valve rings for congestive heart failure: differential durability of repair. Circulation 2006; 114 (1 Suppl): I67-71

19) Silberman S, Klutstein MW, Sabag T, et al: Repair of ischemic mitral regurgitation: comparison between flexible and rigid annuloplasty rings. Ann Thorac Surg 2009; 87: 1721-1726; discussion 17261727

20) Braun J, van de Veire NR, Klautz RJ, et al: Restrictive mitral annuloplasty cures ischemic mitral regurgitation and heart failure. Ann Thorac Surg 2008; 85: 430-436; discussion 436-437

21) Hung J, Papakostas L, Tahta SA, et al: Mechanism of recurrent ischemic mitral regurgitation after annuloplasty: continued LV remodeling as a moving target. Circulation 2004; 110: II85-90

22) Kuwahara E, Otsuji Y, Iguro Y, et al: Mechanism of recurrent/persistent ischemic/functional mitral regurgitation in the chronic phase after surgical annuloplasty: importance of augmented posterior leaflet tethering. Circulation 2006; 114 (1 Suppl): I529-534

23) Magne J, Pibarot P, Dagenais F, et al: Preoperative posterior leaflet angle accurately predicts outcome after restrictive mitral valve annuloplasty for ischemic mitral regurgitation. Circulation 2007; 115: $782-791$

24) Messas E, Bel A, Szymanski C, et al: Relief of mitral leaflet tethering following chronic myocardial infarction by chordal cutting dimin- ishes left ventricular remodeling. Circ Cardiovasc Imaging 2010; 3: 679-686

25) Messas E, Guerrero JL, Handschumacher MD, et al: Chordal cutting: a new therapeutic approach for ischemic mitral regurgitation. Circulation 2001; 104: 1958-1963

26) Messas E, Pouzet B, Touchot B, et al: Efficacy of chordal cutting to relieve chronic persistent ischemic mitral regurgitation. Circulation 2003; 108 (Suppl 1): II111-115

27) Borger MA, Murphy PM, Alam A, et al: Initial results of the chordal-cutting operation for ischemic mitral regurgitation. J Thorac Cardiovasc Surg 2007; 133: 1483-1492

28) Calafiore AM, Refaie R, Iacò AL, et al: Chordal cutting in ischemic mitral regurgitation: a propensity-matched study. J Thorac Cardiovasc Surg 2014; 148: 41-46

29) Hvass U, Tapia M, Baron F, et al: Papillary muscle sling: a new functional approach to mitral repair in patients with ischemic left ventricular dysfunction and functional mitral regurgitation. Ann Thorac Surg 2003; 75: 809-811

30) Rama A, Praschker L, Barreda E, et al: Papillary muscle approximation for functional ischemic mitral regurgitation. Ann Thorac Surg 2007; 84: 2130-2131

31) Kron IL, Green GR, Cope JT: Surgical relocation of the posterior papillary muscle in chronic ischemic mitral regurgitation. Ann Thorac Surg 2002; 74: 600-601

32) Langer F, Schäfers HJ: RING plus STRING: papillary muscle repositioning as an adjunctive repair technique for ischemic mitral regurgitation. J Thorac Cardiovasc Surg 2007; 133: 247-249

33) Arai H, Itoh F, Someya T, et al: New surgical procedure for ischemic/functional mitral regurgitation: mitral complex remodeling. Ann Thorac Surg 2008; 85: 1820-1822

34) Nappi F, Lusini M, Spadaccio C, et al: Papillary muscle approximation versus restrictive annuloplasty alone for severe ischemic mitral regurgitation. J Am Coll Cardiol 2016; 67: 2334-2346

35) Lorusso R, Gelsomino S, Vizzardi E, et al; ISTIMIR Investigators: Mitral valve repair or replacement for ischemic mitral regurgitation? The Italian Study on the Treatment of Ischemic Mitral Regurgitation (ISTIMIR). J Thorac Cardiovasc Surg 2013; 145: 128-139; discussion $137-138$

36) Sharma A, Agrawal S, Goel S, et al: Surgical treatment of ischemic mitral regurgitation: valve repair versus replacement. Curr Cardiol Rep 2017; 19: 3

37) Mihos CG, Santana O: Mitral valve repair for ischemic mitral regurgitation: lessons from the Cardiothoracic Surgical Trials Network randomized study. J Thorac Dis 2016; 8: E94-99

38) Matsui Y, Shingu Y, Wakasa S, et al: Papillary muscle tugging approximation for functional mitral regurgitation. Ann Thorac Surg 2019; doi: 10.1016/j.athoracsur.2018.11.072. [Epub ahead of print]

39) Giannini C, Fiorelli F, De Carlo M, et al: Comparison of percutaneous mitral valve repair versus conservative treatment in severe functional mitral regurgitation. Am J Cardiol 2016; 117: 271-277

40) Mendirichaga R, Singh V, Blumer V, et al: Transcatheter mitral valve repair with mitraclip for symptomatic functional mitral valve regurgitation. Am J Cardiol 2017; 120: 708-715 